\title{
About I, Pernot M, Sudre A (eds) Mondes tsiganes. Une histoire photographique, 1860-1980. Actes Sud/Musée national de l'histoire de l'immigration (2018)
}

\author{
G. Boëtsch
}

(C) Société d'Anthropologie de Paris et Lavoisier SAS 2018

Cet ouvrage-catalogue accompagne l'exposition « Mondes tsiganes » qui a lieu du 13 mars au 26 août 2018 au Musée national de l'histoire de l'immigration (Paris). Les mondes nomades ont toujours constitué un thème de recherche de choix pour l'anthropologie. Parmi ceux-ci, les Tsiganes, romanichels, Gitans, bohémiens constituent des groupes reconnus aujourd'hui sous le vocable générique de Roms. Leurs diverses origines, réelles ou mythiques (Inde, Égypte, Europe centrale, Espagne, etc.), tout comme leur visibilité dans de nombreuses régions d'Europe en ont fait des peuples étranges vivant à proximité, mais pour lesquels notre regard est longtemps demeuré éloigné. Leurs mobilités, leurs modes de vie, tout comme leurs attitudes et leurs visages sont très bien mis en valeur dans ce riche ouvrage d'anthropologie photographique accompagné de textes de qualité. Il s'agit d'un itinéraire présenté sous forme d'un beau et riche ouvrage sur le monde des Roms qui montre à la fois une période disparue - en particulier le travail photographique de Yoors [1] - et la Période moderne allant de la fin de la Seconde Guerre mondiale à nos jours. Si les photographies nous révèlent bien la diversité des histoires et des situations, heureuses et malheureuses, elles montrent aussi comment l'anthropologie s'est précocement emparée du sujet. Jacques-Philippe Potteau, du Muséum d'histoire naturelle va photographier des bohémiens de Paris (1865) tout comme le prince Roland Bonaparte va fixer sur le papier les Gitans d'Espagne (1880-1890) dans son vaste inventaire anthropologico-photographique composé de plus de 200 albums anthropologiques. Les épreuves de Jacques-Philippe Potteau seront présentées par Paul Bataillard à l'Exposition universelle de Paris en 1878 ; exposition

\section{G. Boëtsch $(\square)$}

UMIESS 3189, CNRS, Dakar, Sénégal

CNRS, 3 rue Michel-Ange, 75116, Paris, France

e-mail : boetschgilles@gmail.com où une section anthropologique permit d'observer à la fois des photographies tout comme des crânes d'assassins. L'anthropologue suisse Eugène Pittard participa à des enquêtes ethnographiques et photographiques sur les Tsiganes de Roumanie entre 1899 et 1910 [2]. Exhibés à Paris au Jardin d'Acclimatation en 1913, les « Tcherkesses caucasiens » furent mesurés par un savant de la Société d'anthropologie de Paris [3] et donnèrent lieu à une série de cartes postales éditées par les frères Antonin et Étienne Neurdin à Paris. Ces travaux anthropologiques s'inscrivent dans un vaste travail d'inventaire de la diversité humaine alliant les travaux d'anthropométrie aux photographies. Le biais des travaux du XIX ${ }^{\mathrm{e}}$ siècle était de rechercher l'échantillon le plus représentatif du groupe comme le rappelait Paul Broca dans ses Instructions pour les voyageurs parus en 1860. Dans le cas des Roms, cet ouvrage de photographies montre bien comment la marginalisation et la mise à distance sont associées à l'idée qu'ils constitueraient une population dangereuse. Les clichés judiciaires, un peu systématiques, tout comme les carnets anthropométriques d'identité réservés aux « nomades » confortent cette idée. La partie concernant la Dernière Guerre rappelle le lourd tribut que les Roms ont aussi payé à la barbarie nazie, même si l'iconographie est peu présente. Après 1945, bien que la presse populaire continue à véhiculer les stéréotypes racistes, les situations évoluent, car la modernité frappe aussi à la porte des Roms. Les photographies signent cette transformation. Même si la lecture des lignes de la main, la musique et la danse semblent perdurer, ils représentent un passé en voie de disparition, et la palette des métiers se diversifie, les mobil homes remplacent les roulottes comme les voitures, les chevaux. Certains artistes — comme Django Reinhardt — deviennent des célébrités et peuvent faire connaître au monde entier la culture de leur communauté. Le thème tout comme le parcours présenté dans cet ouvrage en font un sujet ancré aujourd'hui dans notre histoire collective sur lequel l'anthropologie doit faire preuve de réflexivité. 


\section{Références}

1. Yoors J (1990 [1967]) Tsiganes. Phébus, Paris

2. Pittard E (1931) Les Tziganes ou Bohémiens. Recherches anthropologiques dans la péninsule des Balkans. Le Globe (Genève) 70:1-286
3. Bloch A (1913) De l'origine et de l'évolution des peuples du Caucase à propos des Tcherkesses actuellement exhibés au Jardin d'Acclimatation. In: Bulletins et mémoires de la Société d'anthropologie de Paris, VI Série. Tome 4, fascicules 3-4, pp 419-33 